\title{
Perfil das internações hospitalares por doenças do aparelho respiratório no Estado de Sergipe: Uma série histórica
}

\author{
Profile of hospitalizations for respiratory system diseases in the state of Sergipe: A historical series \\ Perfil de hospitalizaciones por enfermedades respiratórias en el estado de Sergipe: Una serie
}

histórica

Recebido: 03/04/2021 | Revisado: 15/04/2021 | Aceito: 20/04/2021 | Publicado: 05/05/2021

Maria Fernanda de Sá Camarço ORCID: https://orcid.org/0000-0002-5012-2968 Universidade Tiradentes, Brasil

E-mail: mfscamarco@gmail.com

Maria Vitória Santos de Jesus ORCID: https://orcid.org/0000-0002-8036-9056 Universidade Tiradentes, Brasil E-mail: vitoria_stos15@hotmail.com

Rebecca Maria Oliveira de Góis ORCID: https://orcid.org/0000-0002-3935-5904 Universidade Tiradentes, Brasil

E-mail: rebecca.gois@ hotmail.com

Patrícia Alves Galhardo Varanda ORCID: https://orcid.org/0000-0001-8120-7983 Universidade Federal da Bahia, Brasil E-mail: pati_ag@yahoo.com.br

Hendyara Oliveira Carvalho Almeida ORCID: https://orcid.org/0000-0003-1923-4766 Universidade Tiradentes, Brasil

E-mail: hendyaracarvalho@hotmail.com

Fernanda Costa Martins Gallotti ORCID: https://orcid.org/0000-0002-9063-1273 Universidade Tiradentes, Brasil E-mail: fercosmart@gmail.com

Fernanda Kelly Fraga Oliveira ORCID: https://orcid.org/0000-0002-9094-6128 Universidade Tiradentes, Brasil

E-mail: fernandadaponte@hotmail.com

Manuela de Carvalho Vieira Martins ORCID: https://orcid.org/0000-0003-1222-5955 Universidade Tiradentes, Brasil

E-mail: manuela.cvm@hotmail.com

Juliana de Oliveira Musse Silva ORCID: https://orcid.org/0000-0003-1713-6791 Universidade Tiradentes, Brasil E-mail: julimusse@hotmail.com

\begin{abstract}
Resumo
Objetivo: Descrever as principais características de distribuição temporal, por faixa etária e sexo, de internações hospitalares por doenças do aparelho respiratório no estado de Sergipe, a partir dos registros de internações no SUS, no período de 2015 a 2019. Metodologia: Trata-se de um estudo ecológico, de abordagem quantitativa, a partir de dados secundários do DATASUS, através da análise das AIHs. Resultados: Identificou-se 33.768 internações, sendo o sexo masculino mais prevalente com $53,6 \%$, enquanto houve $46,4 \%$ internações do sexo feminino. A faixa etária predominante foi de 1 a 4 anos com 8.056 internações. Ainda, 8,9\% internações foram eletivas e 91,1\% de urgência. O maior número de óbitos foi em 2019 com 886 óbitos e, a maior taxa de mortalidade em 2016 com 12,87\%. A média permanência foi maior em 2019, registrando 7,6 dias de internação. Discussão: Verificou-se que Sergipe acompanha os dados nacionais e internacionais a respeito da hospitalização por doenças respiratórias. Na Europa, causam 660.000 mortes e cerca de 6 milhões de internações, contribuindo com 7\% de todas causas de hospitalização, sendo a terceira principal causa de morte. Considerações finais: $\mathrm{O}$ estudo possibilitou conhecer o perfil das internações hospitalares relacionadas a doenças respiratórias em Sergipe, servindo como instrumento de planejamento para estratégias que visam prevenir novos casos e qualificar a assistência.
\end{abstract}

Palavras-chave: Aparelho respiratório; Doenças respiratórias; Epidemiologia; Hospitalização. 


\begin{abstract}
Objective: To describe the main characteristics of temporal distribution, by age group and sex, of hospital admissions for diseases of the respiratory system in the state of Sergipe, based on the records of hospitalizations in the SUS, in the period from 2015 to 2019. Methodology: This is an ecological study, with a quantitative approach, based on secondary data from DATASUS, through the analysis of AIHs. Results: 33,768 hospitalizations were identified, with the male sex being more prevalent with $53.6 \%$, while there were $46.4 \%$ female hospitalizations. The predominant age group was 1 to 4 years with 8,056 hospitalizations. Still, $8.9 \%$ hospitalizations were elective and $91.1 \%$ were urgent. The highest number of deaths was in 2019 with 886 deaths and the highest mortality rate in 2016 with $12.87 \%$. The average stay was higher in 2019, registering 7.6 days of hospitalization. Discussion: It was found that Sergipe follows national and international data regarding hospitalization for respiratory diseases. In Europe, they cause 660,000 deaths and about 6 million hospitalizations, contributing 7\% of all causes of hospitalization, being the third leading cause of death. Final considerations: The study made it possible to know the profile of hospital admissions related to respiratory diseases in Sergipe, serving as a planning tool for strategies aimed at preventing new cases and qualifying assistance.
\end{abstract}

Keywords: Respiratory system; Respiratory tract diseases; Epidemiology; Hospitalization.

\title{
Resumen
}

Objetivo: Revelar las principales características de la distribución temporal, por edad y sexo, de las hospitalizaciones por enfermedades respiratorias en el estado de Sergipe, a partir de dos registros de hospitalizaciones en el SUS, en el período de 2015 a 2019. Metodología: Esta es un estudio ecológico, con enfoque cuantitativo, utilizando datos secundarios de DATASUS, mediante el análisis de HAI. Resultados: se identificaron 33.768 pasantes, siendo hombres o más prevalentes con 53,6\%, mientras que 46,4\% mujeres. El grupo de edad predominante fue de 1 a 4 años con 8.056 internacionales. Además, 8,9\% de escuelas electivas internacionales y $91,1 \%$ de servicios de emergencia. O mayor número de muertes fue en 2019 con 886 muertes y, mayor tasa de mortalidad en 2016 con 12,87\%. La estancia media fue mayor en 2019, registrándose 7,6 días de hospitalización. Discusión: Descubrí que Sergipe sigue datos nacionales e internacionales sobre hospitalizaciones por enfermedades respiratorias. En Europa provocó 660 mil muertes y cerca de 6 mil internacionales, contribuyendo con el 7\% de todas las causas de hospitalización, siendo la tercera causa de muerte. Consideraciones finales: Estudio la posibilidad de conocer el perfil de ingresos hospitalarios relacionados con la educación respiratoria en Sergipe, sirviendo como instrumento para planificar estrategias orientadas a la prevención de nuevos casos y habilitación asistencial.

Palabras clave: Sistema respiratorio, Enfermedades respiratorias, Epidemiología; Hospitalización.

\section{Introdução}

As doenças respiratórias, agudas ou crônicas, estão presentes em todas as faixas etárias e apresentam diversas etiologias e formas de manifestação. As infecções agudas das vias aéreas superiores são as mais conhecidas pela população, incluindo o resfriado, a amigdalite e a sinusite. Destaca-se assim, que podem apresentar complicações ou evoluir para suas formas mais graves, tornando-se necessária a internação hospitalar. Quanto às crônicas, as doenças dasvias aéreas inferiores são as mais frequentes, como a bronquite, o enfisema e a asma. Contudo, quando se trata das doenças respiratórias crônicas, a asma é a principal causa de internação hospitalar no Sistema Único de Saúde (SUS), sendo a bronquite, o enfisema e as outras doenças pulmonares obstrutivas crônicas as principais causas de óbito (Dias, Mendonça, Pinto, Borges, \& Oliveira, 2020) e (Donaldson et al., 2015).

Em relação às infecções agudas de vias aéreas inferiores, a pneumonia é uma das principais causas de morte, representando mais de 4 milhões de óbitos por ano, sendo ela umacausa importante de internações e de mortalidade em países de baixa e média renda como o Brasil (Damasio et al., 2020).

As infecções respiratórias de cunho viral podem ocorrer em epidemias e espalhar-se rapidamente em comunidades em todo o mundo. Nos últimos 20 anos, ocorreram várias epidemias virais, como a Síndrome Respiratória Aguda Grave (SARSCoV) em 2002 a 2003, e a Influenza A (subtipo H1N1) em 2009. Mais recentemente, o coronavírus da Síndrome Respiratória do Oriente Médio (MERS-CoV), identificado pela primeira vez na Arábia Sauditaem 2012 e, atualmente, sendo os primeiros casos reportados em dezembro de 2019, o mundo vive a pandemia causada pelo Novo Conoravírus, causador da COVID-19, também conhecidacomo "Síndrome Respiratória Aguda Grave (SARS-CoV-2), uma doença que apresenta um espectro clínico 
variando de infecções assintomáticas a quadros graves, possuindo alta transmissibilidade, infectividade e letalidade (CarrilloEsper et al., 2018); (Hernández Rodríguez, 2020); (Hui \& Zumla, 2019) e (Vijay \& Perlman, 2016).

A Organização Mundial da Saúde (OMS) declarou a COVID-19 uma pandemia em 11 demarço de 2020, e até o final do dia 26 de setembro, foram confirmados 32.616.929 casos de COVID-19 no mundo. Os Estados Unidos foi o país com o maior número de casos acumulados (7.033.430), seguido pela Índia (5.903.932), Brasil (4.717.991), Rússia (1.136.048) e Colômbia (794.584). Em relação aos óbitos, foram confirmados 989.733 no mundo até o dia 26 de setembro e o Brasil é o segundo país com maior número de óbitos (141.406) por esta doença (Brasil, 2020); (Walker et al., 2020) e (Wang, Wang, Chen, \& Qin, 2020).

No Brasil, as doenças do aparelho respiratório representam um importante desafio paraos serviços de saúde tornandose o segundo principal motivo de internações hospitalares no país, ficando atrás apenas das doenças cardiovasculares, totalizando 5.926.023 hospitalizações entre os anos de 2015 e 2019, sendo o maior número de casos na região Sudeste com 2.174.410 hospitalizações, seguido da região Nordeste com 1.527.701 internações. Quanto aos óbitos, foram registrados 475.462 óbitos no mesmo período, em decorrência de complicações respiratórias (Firs, 2017); (Wang et al., 2020) e (Who, 2018).

Neste contexto, tendo em vista a importância do tema abordado e seu impacto perante a saúde pública, é primordial que sejam criadas estratégias que possibilitem um melhor atendimento às demandas relacionadas a essas patologias e o enfermeiro tem papel importanteno planejamento e implementação dessas estratégias, visto que ele está na assistência da população em todos os níveis de atenção à saúde, assim ele é imprescindível para a prevenção dessas doenças e, consequentemente, redução da hospitalização e morbimortalidade depacientes com doenças respiratórias. Em Sergipe, há poucos estudos capazes de demonstrar amagnitude desse problema, dificultando o conhecimento a respeito do mesmo e, consequentemente, a implementação de ações que visem desde a prevenção ao tratamento dessas doenças no âmbito estadual (Barreto, da Silva, Nomura, de Fátima Lucena, \& de Abreu Almeida, 2020); (Silva, Maia, \& Souza, 2020).

Dessa forma, o conhecimento acerca das internações hospitalares relacionadas a doenças respiratórias, tornase um importante instrumento de planejamento para estratégias que visam tanto prevenir novos casos, como qualificar a assistência prestada ao público acometido, justificando assim a questão norteadora do referido estudo, sendo ela: "Qual o perfilepidemiológico dos pacientes internados no Sistema Único de Saúde (SUS), por doenças do aparelho respiratório no estado de Sergipe, no período de 2015 a 2019?"

Nesse sentido, o presente estudo objetivou descrever as principais características de distribuição temporal, por faixa etária e sexo, de internações hospitalares por doenças do aparelho respiratório em adultos no estado de Sergipe, bem como o tempo de permanência dospacientes nesse ambiente, óbitos, taxa de mortalidade e os custos dos serviços de saúde, a partirdos registros de internações em hospitais do Sistema Único de Saúde, no período de 2015 a 2019, sendo a delimitação deste recorte temporal com vistas a obter as informações mais recentes acerca do impacto causado por estas doenças, de forma a subsidiar o processo de tomada de decisão frente à assistência de enfermagem.

\section{Metodologia}

Trata-se de um estudo do tipo ecológico, de abordagem quantitativa a partir de dados secundários do Departamento de Informática do Sistema Único de Saúde (DATASUS), com o objetivo de conhecer o perfil epidemiológico dos pacientes internados no Sistema Único de Saúde (SUS), por doenças do aparelho respiratório no estado de Sergipe, no período de 2015 a 2019. O estudo ecológico visa o conhecimento a cerca de uma determinada população, do conjunto de indivíduos, não sendo possível reconhecer a particularidade de cada indivíduo, ou seja, nesse tipo de estudo, não existem informações sobre a doença e exposição do indivíduo, mas do grupo populacional como um todo (Mercenas, Ferreira, de Góis, \& Servo, 2020). 
A pesquisa quantitativa se direciona a coleta e análise de dados sobre variáveis, sendo amplamente utilizada em estudos de investigações epidemiológicas. Esse tipo de abordagem pode determinar a força de associação ou correlação entre as variáveis, além da generalização e objetivação dos resultados através de uma mostra que faz inferência a uma população. Ademais, também pode fazer inferências causais que explicam por que as coisas acontecem ou não de uma forma determinada (Esperón, 2017).

Como critérios de inclusão, foram inseridos no estudo os dados disponíveis no DATASUS, com informações acerca das internações hospitalares por doenças respiratórias no estado de Sergipe, além da taxa de mortalidade da doença, número de óbitos, faixa etária mais acometida, sexo, média permanência e gasto dos serviços hospitalares nos últimos 5 anos, visando trazer dados atualizados sobre o perfil das internações provenientes de patologias do trato respiratório. Em relação aos critérios de exclusão, foram retirados da pesquisa dados contidos em boletins epidemiológicos, dados referentes ao ano de 2020 e os dados que antecedem o período do ano de 2014, além de informações a respeito das condições socioeconômicas dos pacientes internados.

A coleta de dados ocorreu durante o período de setembro e outubro de 2020, e as informações referentes às internações hospitalares foram obtidas pelo DATASUS, por meio do Sistema de Informação de Saúde, em Epidemiológicas e Morbidades, no item Morbidade Hospitalar do SUS (SIH/SUS). Em seguida, foi selecionada a opção dados gerais das AIH no estado de Sergipe, que é a localidade onde o estudo foi realizado, entre os anos de 2015 e 2019.

Após a seleção dos dados referentes ao estado, selecionaram-se por meio dos filtros, os períodos dos meses entre janeiro de 2015 e dezembro de 2019, a fim de coletar as informações dos referidos anos, conforme mostra a Figura 1. A partir desse momento, foram realizadas a seleção das internações hospitalares classificadas nas bases da AIH/SUS, conforme o Capítulo X que corresponde às Doenças do Aparelho Respiratório do Código Internacional de Doença (CID) 10 e realizado o cruzamento dos filtros com os dados referentes à: taxa de mortalidade, óbitos, média de permanência, valor dos serviços hospitalares, ano de atendimento, faixa etária e sexo. Desse modo, foram levantadas todas as internações hospitalares correspondentes ao sistema respiratório no período entre janeiro de 2015 e dezembro de 2019.

Figura 1 - Fluxograma da Coleta de Dados.
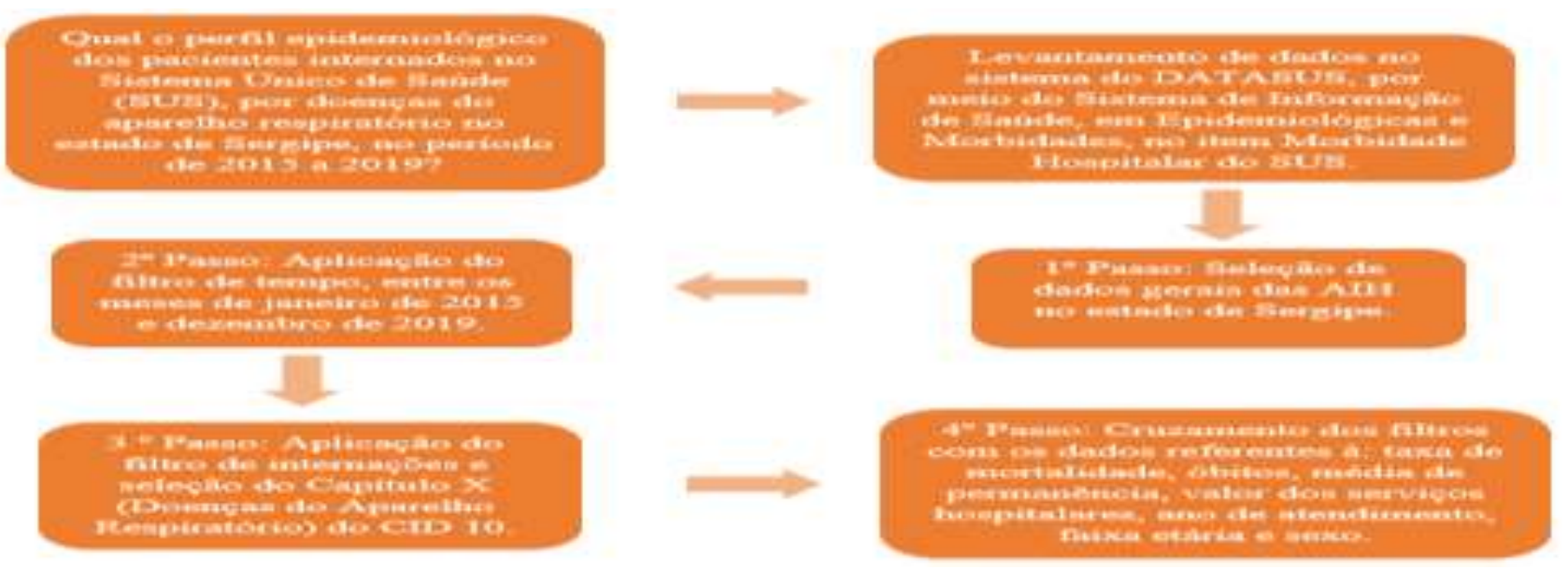

Fonte: Autores.

Os dados do estudo foram obtidos a partir do sistema de informação, sendo este um banco de dados secundários de domínio público, acessíveis e fornecido de modo livre e gratuito, os quais os indivíduos não são identificados. Nesse sentido, não há qualquer possibilidade de dano de ordem física ou moral na perspectiva do indivíduo e das coletividades, por terem 
sidos respeitados os princípios contidos na Resolução No 466, 12/12/2012 do Conselho Nacional de Saúde. Portanto, a presente pesquisa não demandou submissão ao Comitê de Ética em Pesquisa (CEP) da Universidade Tiradentes (UNIT).

\section{Resultados}

Para confecção da Tabela 1, foram selecionados os filtros aplicados nos dados secundários do DATASUS: Internações e Caráter de Atendimento no período de janeiro de 2015 a dezembro de 2019. Durante esse período foram registradas 33.768 internações por doenças respiratórias no estado de Sergipe, sendo que o ano com o maior número de registros foi 2018, apresentando 7.569 (22,4\%) internações, seguido de 2019 com 7.261 (21,5\%), e o período com o menor registro de casos foi 2015 com 5.905 (17,5\%). Quanto ao caráter de atendimento, 3.019 (8,9\%) foram internações eletivas, e $30.749(91,1 \%)$ internações de urgência (Tabela 1).

Tabela 1 - Distribuição das internações por doenças do aparelho respiratório segundo o caráter de atendimento em Sergipe entre os anos de 2015 a 2019.

\begin{tabular}{|c|c|c|c|c|c|c|}
\hline \multirow{3}{*}{$\begin{array}{c}\text { Ano de } \\
\text { Atendimento }\end{array}$} & \multicolumn{2}{|c|}{ Internações } & \multicolumn{4}{|c|}{ Caráter de atendimento } \\
\hline & \multirow[b]{2}{*}{$\mathrm{N}$} & \multirow[b]{2}{*}{$\%$} & \multicolumn{2}{|c|}{ Eletivo } & \multicolumn{2}{|c|}{ Urgência } \\
\hline & & & $\mathrm{N}$ & $\%$ & $\mathrm{~N}$ & $\%$ \\
\hline 2015 & 5.905 & 17,5 & 551 & 1,6 & 5.354 & 15,9 \\
\hline 2016 & 6.300 & 18,7 & 574 & 1,7 & 5.726 & 17,0 \\
\hline 2017 & 6.733 & 19,9 & 722 & 2,1 & 6.011 & 17,8 \\
\hline 2018 & 7.569 & 22,4 & 654 & 1,9 & 6.915 & 20,4 \\
\hline 2019 & 7.261 & 21,5 & 518 & 1,5 & 6.743 & 20,0 \\
\hline Total & 33.768 & 100 & 3.019 & 8,9 & 30.749 & 91,1 \\
\hline
\end{tabular}

Fonte: Ministério da Saúde - Sistema de Informações Hospitalares do SUS (SIH/SUS).

Em uma nova busca, o caráter de atendimento foi substituído pelo filtro Sexo, visando conhecer qual possui mais registros de internações por doenças respiratórias, assim foi possível perceber que o masculino foi o mais prevalente em internações, totalizando 18.090 (53,6\%) durante todo o período, enquanto houve 15.678 (46,4\%) internações provenientes do sexo feminino (Tabela 2). Após os dados referentes ao sexo, buscou-se a faixa etária, sendo que a mais prevalente foi de 1 a 4 anos com 8.056 internações, seguida da faixa etária menor de 1 ano com 5.713 e o menor registros de internação ocorreu na faixa etária de 15 a 19 anos com 776 internações (Tabela 3). 
Tabela 2 - Distribuição das internações por doenças do aparelho respiratório segundo o gênero em Sergipe entre os anos de 2015 a 2019.

\begin{tabular}{ccccccc}
\hline Ano de & \multicolumn{2}{c}{ Internações } & \multicolumn{3}{c}{ Sexo } \\
Atendimento & \multicolumn{2}{c}{ Masculino } & \multicolumn{2}{c}{ Feminino } \\
& $\mathrm{N}$ & $\%$ & $\mathrm{~N}$ & $\%$ & $\mathrm{~N}$ & $\%$ \\
\hline 2015 & 5.905 & 17,5 & 3.184 & 9,4 & 2.721 & 8.1 \\
2016 & 6.300 & 18,7 & 3.323 & 9,8 & 2.977 & 8,8 \\
2017 & 6.733 & 19,9 & 3.564 & 10,6 & 3.169 & 9,4 \\
2018 & 7.569 & 22,4 & 4.125 & 12,2 & 3.444 & 10,2 \\
2019 & 7.261 & 21,5 & 3.894 & 11,5 & 3.367 & 9,9 \\
Total & 33.768 & 100 & 18.090 & 53,6 & 15.678 & 46,4 \\
\hline
\end{tabular}

Fonte: Ministério da Saúde - Sistema de Informações Hospitalares do SUS (SIH/SUS).

Tabela 3 - Distribuição das internações por doenças do aparelho respiratório segundo a faixa etária em Sergipe entre os anos de 2015 a 2019.

\begin{tabular}{ccccccc}
\hline Faixa etária & $\mathbf{2 0 1 5}$ & $\mathbf{2 0 1 6}$ & $\mathbf{2 0 1 7}$ & $\mathbf{2 0 1 8}$ & $\mathbf{2 0 1 9}$ & Total \\
\hline Menor de 1 ano & 1.010 & 1.037 & 969 & 1.464 & 1.233 & 5.713 \\
1 a 4 anos & 1.318 & 1.471 & 1.624 & 1.905 & 1.738 & 8.056 \\
5 a 9 anos & 586 & 622 & 740 & 817 & 769 & 3.534 \\
10 a 14 anos & 234 & 261 & 264 & 286 & 247 & 1.292 \\
15 a 19 anos & 130 & 140 & 177 & 183 & 146 & 1.290 \\
20 a 29 anos & 234 & 242 & 265 & 261 & 288 & 1.230 \\
30 a 39 anos & 231 & 219 & 237 & 276 & 267 & 1.356 \\
40 a 49 anos & 246 & 240 & 282 & 322 & 266 & 1.882 \\
50 a 59 anos & 355 & 370 & 387 & 404 & 366 & 2.414 \\
60 a 69 anos & 418 & 444 & 497 & 487 & 568 & 2.987 \\
70 a 79 anos & 574 & 593 & 631 & 546 & 643 & 3.238 \\
80 anos e mais & 569 & 661 & 660 & 618 & 730 & \\
\hline
\end{tabular}

Fonte: Ministério da Saúde - Sistema de Informações Hospitalares do SUS (SIH/SUS).

Além disso, também foram aplicados os filtros de óbitos, taxa de mortalidade e média permanência, sendo o maior número de óbitos no ano de 2019 com 886 óbitos, seguido do ano de 2016 com 882 mortes e 2015 com o menor número apresentando 737 óbitos. Em relação a taxa de mortalidade, o ano de 2016 registrou a maior taxa (12,87\%) e, o ano que mostrou a menor taxa foi o de 2018 com 9,53\%. A média de permanência foi maior no ano de 2019, registrando uma média de 7,6 dias de internação, sendo que a menor taxa foi no ano de 2017 com uma média de 6,4 dias. 
Os resultados provenientes do levantamento de dados relacionados aos valores dos serviços hospitalares permitem identificar um gasto total superior a 37 milhões de reais, referente a 33.768 internações por doenças do aparelho respiratório no Estado de Sergipe durante o período de janeiro de 2015 a dezembro de 2019.

Ao realizar uma correlação dos dados, percebe-se que os custos com as hospitalizaçõescrescem na medida em que há um maior tempo de permanência, sendo que em 2019 registrou-se a maior média de permanecia $(7,6)$ e, por conseguinte, apresentou maior custo dos serviços ofertados $(8.179 .201,34)$ e, o ano de 2017 apontado com a menor média de permanência $(6,4)$ registrou o menor valor gasto com os serviços $(7.010 .638,48)$.

Tabela 4 - Distribuição das internações por doenças do aparelho respiratório segundo óbitos, taxa de mortalidade, média permanência e valor dos serviços hospitalares em Sergipe entre osanos de 2015 a 2019.

\begin{tabular}{cccccc}
\hline $\begin{array}{c}\text { Ano de } \\
\text { Atendimento }\end{array}$ & \multicolumn{2}{c}{ Óbitos } & $\begin{array}{c}\text { Taxa de } \\
\text { mortalidade(\%) }\end{array}$ & $\begin{array}{c}\text { Média de } \\
\text { permanência(dias) }\end{array}$ & $\begin{array}{c}\text { Valor dos serviços } \\
\text { hospitalares (R\$) }\end{array}$ \\
& $\mathrm{N}$ & $\%$ & & & \\
\hline 2015 & 737 & 18,3 & 11,25 & 6,9 & $7.226 .808,34$ \\
2016 & 882 & 21,9 & 12,87 & 7,0 & $7.096 .704,38$ \\
2017 & 739 & 18,3 & 10,18 & 6,4 & $7.010 .638,48$ \\
2018 & 786 & 19,5 & 9,53 & 6,9 & $7.488 .221,15$ \\
2019 & 886 & 22,0 & 11,02 & 7,6 & $8.179 .201,34$ \\
Total & 4.030 & 100 & 10,97 & - & $37.001 .573,69$ \\
\hline
\end{tabular}

Fonte: Ministério da Saúde - Sistema de Informações Hospitalares do SUS (SIH/SUS).

\section{Discussão}

Segundo D'Aloisio et al. (2019), independente da área geográfica, as doenças respiratórias têm um impacto significativo sobre os gastos com saúde, pois, no mundo, 9 milhões de pessoas morrem de problemas respiratórios a cada ano. $\mathrm{Na}$ Europa, as doenças respiratórias causam 660.000 mortes e são responsáveis por cerca de 6 milhões de internações hospitalares, contribuindo com 7\% de todas as causas de hospitalização. Estima-se que o cenário atual pode piorar em um futuro próximo, visto que as doenças respiratórias são responsáveis por 1/10 da mortalidade geral em todo o mundo, mas espera-se que contribuamcom $1 / 5$ até 2030.

Comparando os dados obtidos através do DATASUS com o estudo de AO et al. (2019), podemos perceber que as crianças com menos de 5 anos e pessoas com 65 anos ou mais de idade têm as maiores taxas de incidência de hospitalização, ou seja, as internações por doenças respiratórias envolveram principalmente as faixas etárias mais vulneráveis, devido ao sistema imunológico comprometido e comorbidades crônicas associadas, assim esse público necessita de uma assistência qualificada visando desde à prevenção ao tratamento dessa doença.

Segundo a Organisation for Economic Co-operation and Development (2018), a mortalidade por doenças respiratórias é a terceira principal causa de morte nos países da UniãoEuropeia (UE), sendo que sua taxa de mortalidade é maior em média $85 \%$ entre os homens doque entre as mulheres em todos os países da UE. Essa taxa elevada se explica devido a uma maior taxa de tabagismo entre os homens, visto que fumar é um importante fator de risco paraessas doenças. Além disso, o diagnóstico em um estágio tardio, contribui para um maior índicede mortalidade. Esse dado acerca da prevalência da mortalidade entre homens 
corrobora com oencontrado neste estudo, onde foi possível perceber que o masculino foi o mais prevalente em internações, totalizando $18.090(53,6 \%)$.

Ainda, a Organisation for Economic Co-operation and Development (2018), traz que muitas mortes por essas patologias podem ser evitadas ao lidar com alguns dos principais fatores de risco e através do aumento da cobertura vacinal para influenza e pneumonia, especialmente entre idosos e outros grupos vulneráveis. O estudo de Khaltaev e Axelrod (2019), corrobora que essas doenças são amplamente evitáveis por meio de políticas públicas que abordem seus fatores de risco como o uso do tabaco, o uso nocivo de álcool, dietas não saudáveis, sedentarismo e poluição do ar.

Para Papaioannou, Bartziokas, Papiris, Loukides, e Kostikas (2016), os pacientes que requerem hospitalização mais prolongada (definida como $\geq 7$ dias) estavam em maior risco de desenvolver exacerbações agudas, sendo que a duração da hospitalização mostrou uma relação estreita com a presença desintomas respiratórios. Já o estudo de Donaldson et al. (2015), mostra que a duração da hospitalização depende das práticas médicas e é distinta da duração dos sintomas. A partir da relação do estudo de Papaioannou et al. (2016), com os dados extraídos do DATASUS referentes à média permanência, percebe-se que no ano de 2019 e de 2016, os pacientes hospitalizados por doenças respiratórias estavam em maior risco de desenvolver exarcebações agudas, visto que é a média, em dias, foi de 7,6 dias de internação no ano de 2019, e de 7,0 dias em 2016.

Em relação à assistência de enfermagem aos portadores de doenças respiratórias, o estudo de Maia et al. (2016), aborda que o enfermeiro, por prestar cuidados diretos e indiretosaos pacientes, é essencial para o reestabelecimento do quadro clínico do doente auxiliando na redução do tempo de internação hospitalar, visando minimizar os riscos de complicação e agravamento da patologia e, por conseguinte, reduzir a taxa de mortalidade das doenças do sistema respiratório. Dada a importância desse profissional para a redução das complicações relacionadas à estas doenças, é primordial que ele participe do delineamento e implementação de ações de saúde que visem a prevenção, o diagnóstico precoce e o tratamento dessas doenças que trazem tantos danos à saúde da população e à saúde pública.

Nesse sentido, de acordo com Barreto et al. (2020), também se faz necessário que as instituições de saúde promovam ações de educações permanentes e instituam protocolos de atendimento para subsidiar a assistência dos profissionais de saúde frente a pacientes com doenças do sistema respiratório, possibilitando a realização do diagnóstico, tratamento e reabilitação dos pacientes, reduzindo as complicações e morbimotalidade por essas doenças. Além disso, essas ações também causarão impacto na diminuição da média permanência hospitalar, bem como na redução dos gastos hospitalares com essas patologias.

\section{Considerações Finais}

O presente estudo possibilitou identificar, a partir dos resultados encontrados, que os dados do estado de Sergipe acompanham os dados nacionais e internacionais a respeito da hospitalização por doenças do sistema respiratório. Além disso, o estudo abordou qual a faixa etária mais acometida por estas patologias, bem como os gastos com essas internações hospitalares, o caráter de atendimento, o número de óbitos e a taxa de mortalidade por ano de atendimento, além de conhecer o sexo mais afetado por estas doenças no estado de Sergipe, entre os anos de 2015 a 2019.

Nesse sentido, a faixa etária mais acometida foi a de 1 a 4 anos com 8.056 internações,sendo o sexo mais prevalente o masculino, a média da taxa de mortalidade dos últimos 5 anos foi de 10,97\%, demonstrando o impacto destas doenças no estado de Sergipe. Além disso, foi gasto um total de R $\$ 37.001 .573,69$ durantes os anos de 2015 a 2019 com serviços para os pacientes internados, demonstrando também a magnitude desse problema de saúde pública para os gastos hospitalares.

Diante do exposto, é primordial que sejam criadas estratégias que possibilitem um melhor atendimento às demandas relacionadas a essas patologias, além da participação do enfermeiro no delineamento e implementação de ações de saúde que visem a prevenção, o diagnóstico precoce e o tratamento dessas doenças que trazem tantos danos à saúde da população e à saúde pública. Além disso, é necessária uma atenção especial aos quadros de doença respiratória em crianças, idosos e em 
pessoas do sexo masculino, pois os índices de hospitalização pelo público supracitado são alarmantes e representam uma parte significativa do número total de casos.

Dessa forma, o presente estudo possibilitou conhecer o perfil das internações hospitalares relacionadas a doenças respiratórias no estado de Sergipe, servindo como um importante instrumento de planejamento para estratégias que visam tanto prevenir novos casos, como qualificar a assistência prestada ao público acometido.

Assim, como perspectiva de trabalhos futuros, recomenda-se ampliar o estudo à nível nacional, além de analisar as principais doenças respiratórias que causam a hospitalização de crianças e idosos, sendo esse público o mais acometido por este problema. Além disso, também faz-se necessários novos estudos que abordem o conhecimento acerca das internações hospitalares relacionadas a doenças respiratórias, bem como a magnitude deste problema impacto perante a saúde pública.

\section{Referências}

Ao, T., McCracken, J. P., Lopez, M. R., Bernart, C., Chacon, R., Moscoso, F., \& Bryan, J. P. (2019). Hospitalization and death among patients with influenza, Guatemala, 2008-2012. BMC public health, 19(3), 463.

Barreto, L. N. M., da Silva, M. B., Nomura, A. T. G., de Fátima Lucena, A., \& de Abreu Almeida, M. (2020). Evolução clínica dos indicadores de resultados de enfermagem em pacientes com padrão respiratório ineficaz. Revista Eletrônica de Enfermagem, 22.

BRASIL. (2020). Boletim Epidemiológico Especial: Doença pelo Coronavírus COVID-19, Semana Epidemiológica 39 (20 a 26/09/2020). Brasília, DF: Ministério da Saúde - Secretariade Vigilância em Saúde.

Carrillo-Esper, R., Vázquez-De Anda, G. F., Mejía-Pérez, C. I., Delaye-Aguilar, M. G., Pérez-Castañeda, A. I., Briones-Garduño, J. C., \& León-Ponce, M. A. D. (2018). At 50 years of the description of acute respiratory distress syndrome. Gaceta medica de Mexico, 154(2), $236-253$.

D’Aloisio, F., Vittorini, P., Giuliani, A. R., Scatigna, M., Del Papa, J., Muselli, M., \& Fabiani, L. (2019). Hospitalization Rates for Respiratory Diseases After L'Aquila Earthquake. International journal of environmental research and public health, 16(12), 2109.

Damasio, G. A., Pereira, L. A., Moreira, S. D., Duarte dos Santos, C. N., Dalla-Costa, L. M., \& Raboni, S. M. (2015). Does virus-bacteria coinfection increase the clinical severity of acute respiratory infection?. Journal of medical virology, 87(9), 1456-1461.

Dias, F. L. T., Mendonça, F. D., Pinto, G. M., Borges, I. S. C., \& de Oliveira, S. V. (2020). Doenças respiratórias no Triângulo Mineiro: Análise epidemiológica e projetiva com a pandemia de COVID-19. Journal of Health \& Biological Sciences, 8(1), 1-6.

Donaldson, G. C., Law, M., Kowlessar, B., Singh, R., Brill, S. E., Allinson, J. P., \& Wedzicha, J. A. (2015). Impact of prolonged exacerbation recovery in chronic obstructive pulmonary disease. American journal of respiratory and critical care medicine, 192(8), 943-950.

Esperón, J. M. T. (2017). Pesquisa quantitativa na ciência da enfermagem. Escola Anna Nery, 21(1).

Foro de Sociedades Respiratorias Internacionales - FIRS (2017). El impacto global de la Enfermedad Respiratoria. Ciudad de México: Asociación Latinoamericana de Tórax.

Hernández Rodríguez, J. (2020). Aspectos clínicos relacionados con el Síndrome Respiratorio Agudo Severo (SARS-CoV-2). Revista Habanera de Ciencias Médicas, 19.

Hui, D. S., \& Zumla, A. (2019). Severe acute respiratory syndrome: historical, epidemiologic, and clinical features. Infectious Disease Clinics, 33(4), 869-889.

Khaltaev, N., \& Axelrod, S. (2019). Tendências globais de mortalidade por doenças respiratórias crônicas, diretrizes de tratamento, modificações no estilo de vida e poluição do ar: análise preliminar. Journal of thoracic disease , 11 (6), 2643.

Maia, L. F. S., dos Santos, B. L., de Jesus Pereira, C. M., Santos, M. M., Rodrigues, E. P., \& dos Santos, M. D. C. (2016). Assistência de enfermagem ao adulto com agravos respiratórios. Revista Recien-Revista Científica de Enfermagem, 6(18), 85-91.

Mercenas, S. L. G., Ferreira, T. P. O., de Góis, R. M. O., \& Servo, M. L. S. (2020). Caracterização das Internações Hospitalares deIdosos no SUS em Sergipe: Estudo Epidemiológico Descritivo do Ano de 2018. Interfaces Científicas-Saúde e Ambiente, 8(2), 9-22.

OECD| European Union. (2018). Health at a Glance: Europe 2018 State of Health in the EU Cycle. OECD.

Papaioannou, AI, Bartziokas, K., Papiris, S., Loukides, S., \& Kostikas, K. (2016). Duração da hospitalização e deterioração da função pulmonar como preditores de futuras exacerbações da doença pulmonar obstrutiva crônica. Jornal americano de medicina respiratória e de cuidados intensivos , 193 (6), 698700 .

Silva, A. P. D. S. C., Maia, L. T. D. S., \& Souza, W. V. D. (2020). Síndrome Respiratória Aguda Grave em Pernambuco: comparativo dos padrões antes e durante a pandemia de COVID-19. Ciência \& Saúde Coletiva, 25, 4141-4150.

Vijay, R., \& Perlman, S. (2016). Middle East respiratory syndrome and severe acute respiratory syndrome. Current opinion in virology, 16, $70-76$.

Walker, P., Whittaker, C., Watson, O., Baguelin, M., Ainslie, K., Bhatia, S., \& Ghani, A. (2020). Relatório 12: O impacto global do COVID-19 e estratégias de mitigação e supressão. 
Research, Society and Development, v. 10, n. 5, e25110513522, 2021

(CC BY 4.0) | ISSN 2525-3409 | DOI: http://dx.doi.org/10.33448/rsd-v10i5.13522

Wang, Y., Wang, Y., Chen, Y., \& Qin, Q. (2020). Unique epidemiological and clinical features of the emerging 2019 novel coronavirus pneumonia (COVID-19) implicate special control measures. Journal of medical virology, 92(6), 568-576.

WHO, W. (2018). The top 10 causes of death. World Health Organization. 